OPEN ACCESS

Edited by:

Haike Antelmann

Freie Universität Berlin, Germany

Reviewed by:

Michael Gänzle,

University of Alberta, Canada Andrew James McBain,

The University of Manchester,

United Kingdom

*Correspondence:

Haiyue Yu

haiyue.yu@charite.de

Specialty section:

This article was submitted to Microbial Physiology and Metabolism,

a section of the journal

Frontiers in Microbiology

Received: 05 March 2020 Accepted: 04 June 2020

Published: 26 June 2020

Citation:

Yu H, Ganas $P$ and

Schwendicke F (2020)

Environment-Specific Probiotic

Supernatants Modify the Metabolic Activity and Survival of Streptococcus

mutans in vitro.

Front. Microbiol. 11:1447.

doi: 10.3389/fmicb.2020.01447

\section{Environment-Specific Probiotic Supernatants Modify the Metabolic Activity and Survival of Streptococcus mutans in vitro}

\author{
Haiyue Yu*, Petra Ganas and Falk Schwendicke
}

Charité-Universitätsmedizin Berlin, corporate member of Freie Universität Berlin, Humboldt-Universität zu Berlin, and Berlin Institute of Health, Department of Oral Diagnosis, Digital Health and Health Services Research, Berlin, Germany

A range of studies showed probiotics like Streptococcus oligofermentans and Limosilactobacillus reuteri to inhibit the cariogenic activity and survival of Streptococcus mutans, possibly via the production of substances like $\mathrm{H}_{2} \mathrm{O}_{2}$, reuterin, ammonia and organic acids. We aimed to assess the environment-specific mechanisms underlying this inhibition. We cultured $L$. reuteri and $S$. oligofermentans in various environments; minimal medium (MM), MM containing glucose ( $M M+G l u)$, glycerol (MM+Gly), lactic acid (MM+Lac), arginine (MM+Arg) and all four substances (MM+all) in vitro. Culture supernatants were obtained and metabolite concentrations (reuterin, ammonia, $\mathrm{H}_{2} \mathrm{O}_{2}$, lactate) measured. $\mathrm{S}$. mutans was similarly cultivated in the above six different $\mathrm{MM}$ variation media, with glucose being additionally added to the $\mathrm{MM}+\mathrm{Gly}, \mathrm{MM}+\mathrm{Lac}$, and MM+Arg group, with (test groups) and without (control groups) the addition of the supernatants of the described probiotic cultures. Lactate production by $S$. mutans was measured and its survival (as colony-forming-units/mL) assessed. $L$. reuteri environment-specifically produced reuterin, $\mathrm{H}_{2} \mathrm{O}_{2}$, ammonia and lactate, as did S. oligofermentans. When cultured in S. oligofermentans supernatants, lactate production by $S$. mutans was significantly reduced $(p<0.01)$, especially in $\mathrm{MM}+\mathrm{Lac}+\mathrm{Glu}$ and $\mathrm{MM}+$ all, with no detectable lactate production at all (controls means \pm SD: $4.46 \pm 0.41 \mathrm{mM}$ and $6.00 \pm 0.29 \mathrm{mM}$, respectively, $p<0.001)$. A similar reduction in lactate production was found when $S$. mutans was cultured in $L$. reuteri supernatants $(p<0.05)$ for all groups except MM+Lac+Glu. Survival of S. mutans cultured in S. oligofermentans supernatants in MM+Lac+Glu and MM+all was significantly reduced by $0.6-\log _{10}$ and $0.5-\log _{10}$, respectively. Treatment with the supernatant of $L$. reuteri resulted in a reduction in the viability of $S$. mutans in $\mathrm{MM}+$ Gly+Glu and $M M+$ all by $6.1-\log _{10}$ and $7.1-\log _{10}$, respectively. Probiotic effects on the metabolic activity and survival of $S$. mutans were environment-specific through different pathways.

Keywords: caries, colony forming units, dental, lactate production, metabolites, probiotics 


\section{INTRODUCTION}

The human oral cavity harbors more than 700 microbial species, which constitute a dynamic microbial community (Aas et al., 2005). The coexistence and competition between different species are central to the oral microbial homeostasis (Bao et al., 2015). A disturbance in this homeostasis, termed dysbiosis, is associated with dental diseases like dental caries or periodontitis (Exterkate et al., 2010). For dental caries, the dominance of acidogenic and aciduric species like Streptococcus mutans, triggered by the abundant intake of fermentable carbohydrates, is associated with a net mineral loss from dental hard tissues and the formation of a caries lesion (Marsh, 2006).

Contemporary caries management aims to rebuild a healthy microbial equilibrium within the dental biofilm (Marsh, 2006; Bao et al., 2015). One strategy supposedly supporting such rebalancing of the biofilm is the application of probiotics. Probiotics are microorganisms, mainly bacteria, that when administered in sufficient amounts, provide health benefits to the host (Ng et al., 2009; Bosch et al., 2012), for example by inhibiting the metabolic activity and survival of harmful microbiota as well as modulating the host's immune response, thereby helping to stabilize the local microecosystem (Meurman, 2005). Probiotics have been tested both in vitro and in clinical studies for their anticaries effect, with mixed results (Jalasvuori et al., 2012; Gruner et al., 2016).

Certain probiotics have been tested more widely. Streptococcus oligofermentans, a synonym of Streptococcus cristatus (Jensen et al., 2016) was isolated from healthy tooth surfaces (Tong, 2003) and has anti-bacterial effects against pathogens like S. mutans (Liu et al., 2014). It produces hydrogen peroxide $\left(\mathrm{H}_{2} \mathrm{O}_{2}\right)$ from lactic acid (Tong et al., 2007, 2008) as well as ammonia from arginine, which may both reduce the amount of free lactic acid, thereby increasing the local $\mathrm{pH}$ and preventing the initiation of a caries lesion or slowing down or stopping lesion progression (Burne and Marquis, 2000; Clancy et al., 2000). Limosilactobacillus reuteri (Zheng et al., 2020) is an obligate heterofermentative probiotic and most strains in its human lineages have the ability to excrete reuterin ( $\mathrm{Mu}$ et al., 2018), a potent antibiotic substance, which exhibits broadspectrum antimicrobial effect on Gram-positive and Gramnegative bacteria (Talarico and Dobrogosz, 1989; Doleyres et al., 2005). In addition to reuterin, $L$. reuteri also produces $\mathrm{H}_{2} \mathrm{O}_{2}$, organic acid (Kang et al., 2011) and ammonia (Mu et al., 2018; Zaura and Twetman, 2019), with possible impact on S. mutans metabolic activity and survival. Furthermore, some strains of L. reuteri generate a unique antagonistic activity, reutericyclin, which shows a broad inhibitory spectrum but has no effect on the growth of gram-negative bacteria (Ganzle et al., 2000; Lin et al., 2015).

The antibacterial effect of these probiotics hence relies, at least in parts, on the production of the described substances. This production, in turn, is likely to be dependent on the environmental conditions, especially the availability of certain educts required to produce reuterin, $\mathrm{H}_{2} \mathrm{O}_{2}$ etc., So far, it was not studied if different environments modify the probiotic effects on cariogenic pathogens like S. mutans. Deeper knowledge on such environmental requirements is needed both for future research (setting up appropriate models considering these requirements) and for clinical applications. For example, it may be feasible to boost the probiotic anti-caries effect by supplementing probiotic products with certain substances required for a specific probiotic activity.

We aimed to assess the environment-specific activity and impact of two different probiotics, S. oligofermentans, and L. reuteri, on the metabolic activity and survival of S. mutans. We hypothesized that the metabolic activity and survival of $S$. mutans is significantly reduced when exposed to probiotic supernatants, and that this effect is environment-specific.

\section{MATERIALS AND METHODS}

This study used an established in vitro model (Ganas and Schwendicke, 2019) to assess the environment-specific impact of probiotics on metabolic activity and survival of $S$. mutans. Different environmental conditions were simulated by using determined modifications of a saliva analog, allowing to deterministically vary the metabolic activity of the two different probiotics, $S$. oligofermentans, and $L$. reuteri. The supernatants resulting from the cultivation of probiotics in different environments were then used to assess their impact on S. mutans metabolic activity, measured via determining the lactate production, and survival, measured via the colonyforming-units $/ \mathrm{mL}$ of $S$. mutans. Controls of $S$. mutans cultured in different environments, but without probiotic supernatant, were additionally used. All assays and tests were performed in three biological replications, each with two technical replications (measurements) whose average was used for statistical analysis.

\section{Bacterial Strains and Growth Conditions}

Three bacterial strains S. mutans, DSM 20523, S. oligofermentans, DSM 8249 (DMSZ, Braunschweig, Germany) and L. reuteri, ATCC PTA 5289 (BioGaia, Stockholm, Sweden) were used. The strains S. mutans and S. oligofermentans were grown on blood agar plates COLS+ (Oxoid, Wesel, Germany) while the strain L. reuteri was maintained on deMan-Rogosa-Sharpe (MRS) agar (Oxoid) at $37^{\circ} \mathrm{C}$ aerobically for $1-2$ days.

\section{Preparation of Deproteinized Supernatants From Probiotics Cultures}

The two probiotics were precultured separately in brainheart-infusion (BHI) broth (Carl Roth, Karlsruhe, Germany) supplemented with $1 \%$ glucose (Carl Roth), $4 \mathrm{~g} / \mathrm{L}$ yeast extract and $8 \mathrm{~g} / \mathrm{L}$ beef extract (Carl Roth) for $18 \mathrm{~h}$ under aerobic conditions at $37^{\circ} \mathrm{C}$ in $15 \mathrm{ml}$ Falcon tubes (Corning, Kaiserslautern, Germany). After centrifugation at $7100 \mathrm{~g}$ for $15 \mathrm{~min}$ at room temperature, the supernatants were removed and the cells were transferred to $0.9 \%$ sodium chloride with an inoculum (means $\pm \mathrm{SD}$ ) of $3.96 \pm 0.37 \times 10^{7}$ cells $/ \mathrm{mL}$ for S. oligofermentans and $4.16 \pm 0.22 \times 10^{7}$ cells $/ \mathrm{mL}$ for L. reuteri, with a total of $6 \mathrm{ml}$-cultures in $15 \mathrm{ml}$ Falcon tubes (Corning), respectively. After another centrifugation at $7100 \mathrm{~g}$ 
for $15 \mathrm{~min}$, the supernatants were discarded and $6 \mathrm{~mL}$ Minimal Medium (MM) was added.

The minimal medium was based on a chemically defined saliva analog (Wong and Sissions, 2001) and consisted of $10 \mathrm{mM}$ $\mathrm{KH}_{2} \mathrm{PO} 4$ (Merck, Darmstadt, Germany), 10 mM K ${ }_{2} \mathrm{HPO} 4$ (Carl Roth), $1 \mathrm{mM} \mathrm{NaCl}$ (Carl Roth), $3 \mathrm{mM} \mathrm{KCl}$ (Merck), $0.2 \mathrm{mM}$ $\mathrm{NH}_{4} \mathrm{Cl}$ (Merck), and $0.2 \mathrm{mM} \mathrm{MgCl}_{2} \times 6 \mathrm{H}_{2} \mathrm{O}$ (Merck). The $\mathrm{MM}$ was supplemented as follows to generate six different MM variations, allowing to assess the relevance of the metabolic environment on the probiotic activity and its association with the inhibition of $S$. mutans survival and activity: (1) MM, (2) MM with $5 \mathrm{mM}$ glucose (Carl Roth) (MM+Glu), (3) MM with $300 \mathrm{mM}$ glycerol (Sigma-Aldrich, Taufkirchen, Germany) (MM+Gly), (4) MM with $5 \mathrm{mM}$ Lactic acid (Carl Roth) (MM+Lac), (5) $\mathrm{MM}$ with $5 \mathrm{mM}$ arginine (Sigma-Aldrich) (MM+Arg) and (6) MM with $5 \mathrm{mM}$ glucose, $300 \mathrm{mM}$ glycerol, $5 \mathrm{mM}$ Lactic acid and $5 \mathrm{mM}$ arginine $(\mathrm{MM}+\mathrm{all})$. The final $\mathrm{pH}$ of the six MM variations was MM 6.95, MM+Glu 6.90, MM+Gly 6.92, $\mathrm{MM}+\mathrm{Lac}$ 6.61, MM+Arg 7.14, MM+all 6.83. For the culture of $S$. mutans, in order to enable it to produce lactate, glucose was added to $\mathrm{MM}+\mathrm{Gly}, \mathrm{MM}+\mathrm{Lac}, \mathrm{MM}+\mathrm{Arg}$ group to a final concentration of $5 \mathrm{mM}$.

In a pre-experiment, the cultivation period of the probiotics was varied (15 $\mathrm{min}, 30 \mathrm{~min}, 2,4$, and $18 \mathrm{~h}$ ) to gauge the impact of this period on the production of $\mathrm{H}_{2} \mathrm{O}_{2}$, lactate, reuterin and ammonia. Different probiotic cultivation periods were eventually used to generate supernatants optimally enriched with these metabolites. As a consequence, S. oligofermentans, was cultured in $\mathrm{MM}+\mathrm{Glu}$ and $\mathrm{MM}+\mathrm{Lac}$ aerobically at $37^{\circ} \mathrm{C}$ for $30 \mathrm{~min}$, in $\mathrm{MM}$ and $\mathrm{MM}+\mathrm{Gly}$ for $2 \mathrm{~h}$, and in $\mathrm{MM}+\mathrm{Arg}$ and $\mathrm{MM}+$ all for $4 \mathrm{~h}$, followed by centrifugation at $20800 \mathrm{~g}$ for $10 \mathrm{~min}$ at room temperature to obtain the supernatants. L. reuteri was cultured in $\mathrm{MM}+\mathrm{Glu}, \mathrm{MM}+\mathrm{Gly}, \mathrm{MM}+\mathrm{Lac}$ and $\mathrm{MM}+$ all for $4 \mathrm{~h}$ and in $\mathrm{MM}$ and $\mathrm{MM}+\mathrm{Arg}$ for $18 \mathrm{~h}$, followed by the same protocol to collect supernatants. Deproteinization was conducted using Amicon Ultra-2ml centrifugal filter units with molecular weight cut-off (MWCO) of $10 \mathrm{kDa}$ (Merck) at $7500 \mathrm{~g}$ for $20 \mathrm{~min}$ at room temperature. The deproteinized supernatants were maintained at $-80^{\circ} \mathrm{C}$ for later processing. Control samples without bacteria were established using BHI medium containing $1 \%$ Glucose, $4 \mathrm{~g} / \mathrm{L}$ yeast extract and $8 \mathrm{~g} / \mathrm{L}$ beef extract followed by six different $\mathrm{MM}$ variations treated in the same way as in the bacterial culture groups.

\section{Preparation of Deproteinized Supernatants From S. mutans Cultures}

S. mutans was precultured in the $\mathrm{BHI}+1 \%$ glucose $+4 \mathrm{~g} / \mathrm{L}$ yeast extract $+8 \mathrm{~g} / \mathrm{L}$ beef extract medium for $18 \mathrm{~h}$ aerobically at $37^{\circ} \mathrm{C}$ in $15 \mathrm{~mL}$ Falcon tubes. After centrifugation at $7100 \mathrm{~g}$ for $15 \mathrm{~min}$ at room temperature, cultivation supernatants were removed. Cells were rinsed with $0.9 \%$ sodium chloride and after another centrifugation at $7100 \mathrm{~g}$ for $15 \mathrm{~min}$, bacteria were transferred to $\mathrm{MM}, \mathrm{MM}+\mathrm{Glu}, \mathrm{MM}+\mathrm{Gly}+\mathrm{Glu}, \mathrm{MM}+\mathrm{Lac}+\mathrm{Glu}, \mathrm{MM}+\mathrm{Arg}+\mathrm{Glu}$ and $\mathrm{MM}+$ all media, inoculated with $4.35 \pm 0.39 \times 10^{7}$ cells as $1 \mathrm{~mL}$-cultures in $1.5 \mathrm{~mL}$ Eppendorf tubes (Eppendorf, Hamburg,
Germany), followed by incubation at $37^{\circ} \mathrm{C}$ aerobically for further $18 \mathrm{~h}$. Control samples without bacteria were established using BHI medium containing $1 \%$ Glucose, $4 \mathrm{~g} / \mathrm{L}$ yeast extract and $8 \mathrm{~g} / \mathrm{L}$ beef extract followed by six different $\mathrm{MM}$ variations treated in the same way as in the bacterial culture groups, except that $5 \mathrm{mM}$ glucose was additionally added to MM+Gly, MM+Lac, MM+Arg groups. Afterward, the deproteinized supernatants were collected and stored as described above.

\section{Culture of S. mutans With Probiotic Supernatants}

After pre-incubation in $\mathrm{BHI}+1 \%$ glucose $+4 \mathrm{~g} / \mathrm{L}$ yeast extract $+8 \mathrm{~g} / \mathrm{L}$ beef extract medium for $18 \mathrm{~h}, \mathrm{~S}$. mutans was treated in the same manner as above with an inoculum of $4.29 \pm 0.65 \times 10^{7}$ cells $/ \mathrm{mL}$. After centrifugation, the supernatants were discarded and $1 \mathrm{~mL}$ of the supernatants of S. oligofermentans and L. reuteri (cultured in the different $\mathrm{MM}$ as described) were pipetted into $1.5 \mathrm{~mL}$ Eppendorf tubes (Eppendorf). Glucose was additionally added to the $\mathrm{MM}+\mathrm{Gly}, \mathrm{MM}+\mathrm{Lac}$, and MM+Arg at a final concentration of $5 \mathrm{mM}$. S. mutans was cultured for further $18 \mathrm{~h}$ as before. The probiotic supernatant without bacteria was used as control. The deproteinized supernatants of S. mutans were collected and stored as described above.

\section{Metabolite Assays}

The metabolite production of lactate, $\mathrm{H}_{2} \mathrm{O}_{2}$ and ammonia was measured via assessing their concentration in the deproteinized supernatants using colorimetric assay kits (Sigma-Aldrich) in accordance with the manufacturers' instructions. The determination of reuterin in the supernatants was analyzed as described elsewhere (Kang et al., 2011) with some modifications. In short, $250 \mu \mathrm{L}$ of deproteinized probiotic supernatant samples were added to $187.5 \mu \mathrm{L}$ of $10 \mathrm{mM}$ tryptophan dissolved in 0.05 $\mathrm{M} \mathrm{HCl}$, followed by $750 \mu \mathrm{L}$ of $37 \% \mathrm{HCl}$. Under acidic conditions, tryptophan and the aldehyde of reuterin form a $\beta$-carboline derivative which oxidizes to produce a purple pigment. After incubation at $37^{\circ} \mathrm{C}$ for $20 \mathrm{~min}$, the absorbance was measured at $560 \mathrm{~nm}$. Acrolein (Sigma) was used as the calibration standard. To obtain standard curve, $0-15 \mu \mathrm{mol}$ of acrolein was added to $1 \mathrm{~mL}$ of distilled water. The detection of absorbance was performed by the 96 well plate spectrophotometer Multiskan Go (Thermo Fisher Scientific, Schwerte, Germany).

\section{Viable Bacteria Enumeration}

Viable bacteria cells were determined by plating $100 \mu \mathrm{L}$ aliquots of $1 \mathrm{~mL}$ serial dilutions on COLS+ agar plates for S. oligofermentans and $S$. mutans or on MRS agar plates for L. reuteri. After 1-2 days of aerobic incubation at $37^{\circ} \mathrm{C}$, the colony forming units/mL $(\mathrm{CFU} / \mathrm{mL})$ were calculated.

\section{Statistical Analysis}

Descriptive analysis was performed, and one-way ANOVA followed by Dunnett's test conducted, with $P<0.05$ considered as statistically significant. SPSS Version 20.0 software (SPSS Inc., Chicago, IL, United States) was used for statistical analysis. 


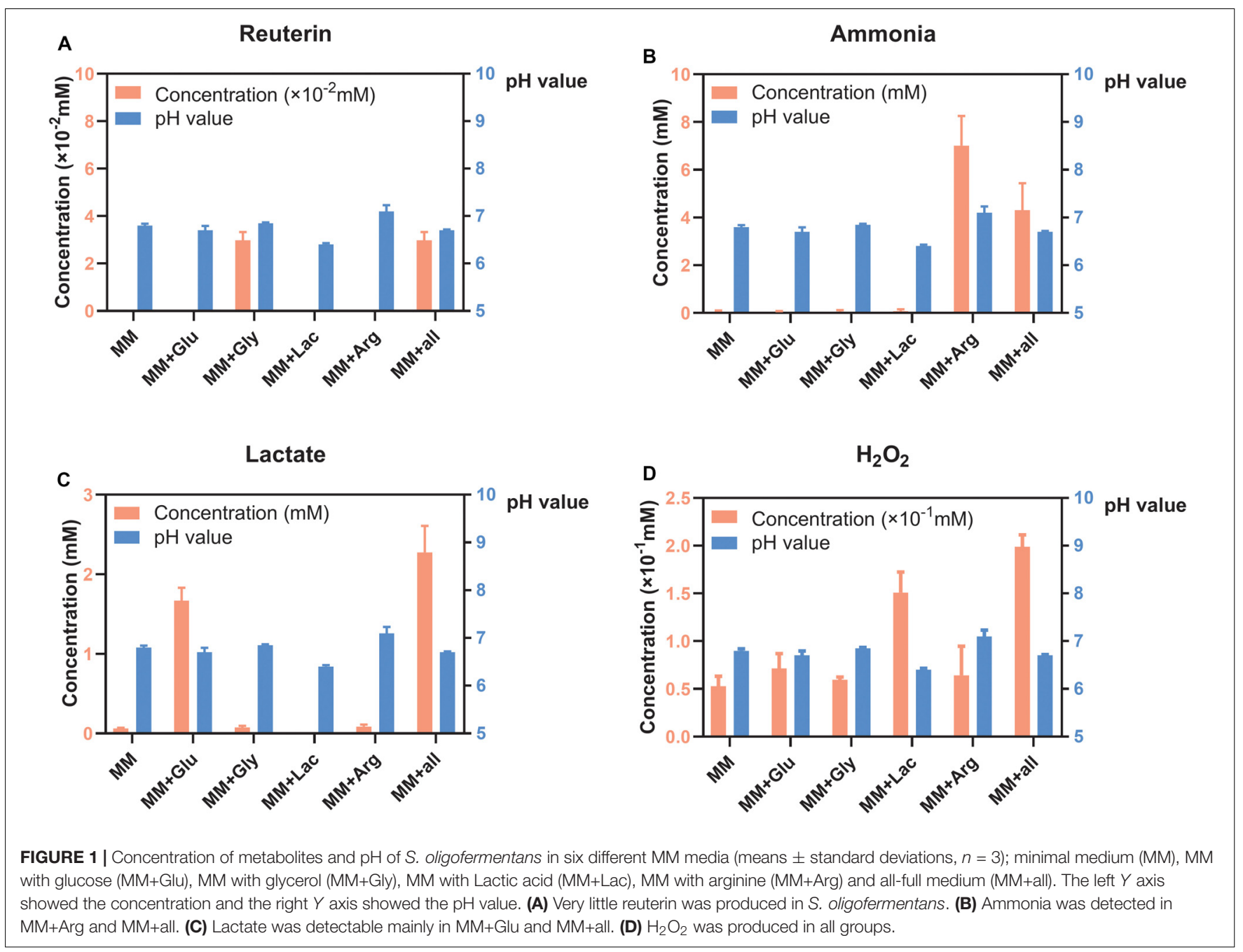

\section{RESULTS}

\section{Concentration of Metabolites of S. oligofermentans Under Different Environmental Conditions}

S. oligofermentans produced very little reuterin in MM+Gly and $\mathrm{MM}+$ all (Figure 1A). Ammonia was detected in $\mathrm{MM}+\mathrm{Arg}$ and $\mathrm{MM}+$ all (Figure 1B) and lactate was detectable mainly in $\mathrm{MM}+\mathrm{Glu}$ and $\mathrm{MM}+$ all (Figure $1 \mathrm{C}$ ). $\mathrm{H}_{2} \mathrm{O}_{2}$ was produced in all groups, with the highest concentration in $\mathrm{MM}+$ all (Figure 1D). The $\mathrm{pH}$ of the supernatants after the incubation of S. oligofermentans were MM 6.80, MM+Glu 6.70, MM+Gly 6.85, $\mathrm{MM}+\mathrm{Lac}$ 6.40, MM+Arg 7.10, MM+all 6.70 (Figure 1).

\section{Concentration of Metabolites of \\ $L$. reuteri in Six Different MM Medium}

Reuterin was detected in both $\mathrm{MM}+\mathrm{Gly}$ and $\mathrm{MM}+$ all (Figure 2A), while ammonia was only detected in $\mathrm{MM}+\mathrm{Arg}$ (Figure 2B). Lactate was detected in $\mathrm{MM}+\mathrm{Glu}$ and $\mathrm{MM}+$ all, with only very low concentrations in the other groups (Figure 2C).
$\mathrm{H}_{2} \mathrm{O}_{2}$ was produced in all groups, the highest concentration being measured in $\mathrm{MM}+$ all (Figure 2D). The $\mathrm{pH}$ of the supernatants after the incubation of $L$. reuteri were MM 6.85, $\mathrm{MM}+\mathrm{Glu}$ 6.80, MM+Gly 6.80, MM+Lac 6.40, MM+Arg 7.20, $\mathrm{MM}+$ all 6.60 (Figure 2).

\section{Effect of Probiotic Supernatants on S. mutans Metabolic Activity}

Lactate production of $S$. mutans was minimal $\left(1.78 \pm 0.65 \times 10^{-2} \mathrm{mM}\right)$ in control medium without glucose, and significantly higher in control media with glucose. Cultivation in supernatant of $S$. oligofermentans significantly reduced the lactate production of $S$. mutans regardless of the medium (Table 1) and, via utilization of lactate by $S$. mutans, even decreased the concentration of lactate in $\mathrm{MM}+$ all (concentration decreased by $-2.14 \pm 0.35 \mathrm{mM}$ ) and $\mathrm{MM}+\mathrm{Lac}+\mathrm{Glu}$ (concentration decreased by $-1.39 \pm 0.25 \mathrm{mM}$ ) respectively, $(p<0.001)$. Cultivation in supernatant of $L$. reuteri also significantly reduced the lactate production of $S$. mutans in all media except MM+Lac+Glu (Table 1). 
A

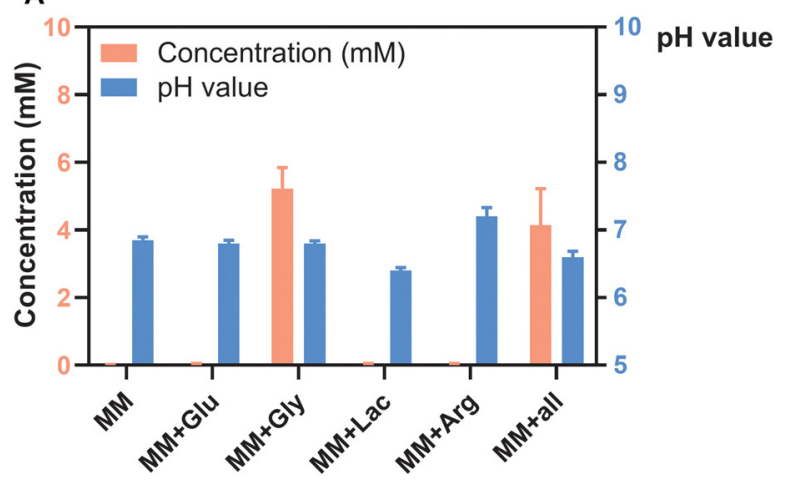

C

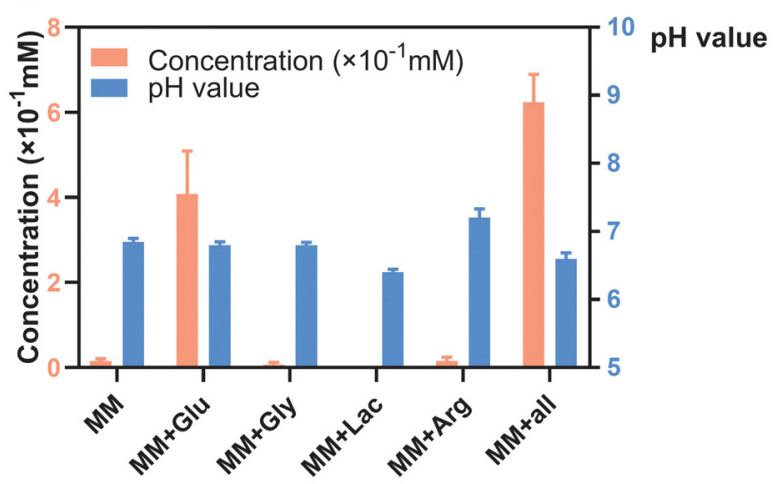

B
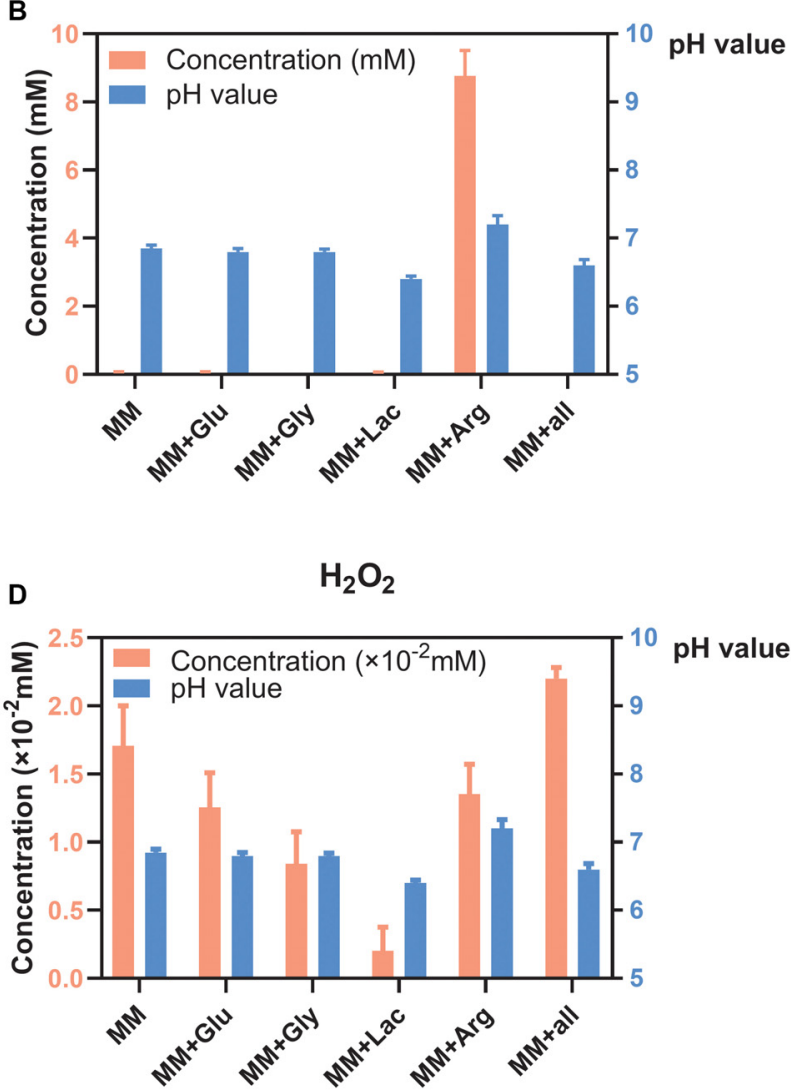

FIGURE 2 | Concentration of metabolites and pH of $L$. reuteri (means \pm standard deviations, $n=3$ ) in six different MM media; minimal medium (MM), MM with glucose (MM+Glu), MM with glycerol (MM+Gly), MM with Lactic acid (MM+Lac), MM with arginine (MM+Arg) and all-full medium (MM+all). The left $Y$ axis showed the concentration and the right $Y$ axis showed the $\mathrm{pH}$ value. (A) Reuterin was detected in both $\mathrm{MM}+\mathrm{Gly}$ and $\mathrm{MM}+$ all. (B) Ammonia was only detected in $\mathrm{MM}+\mathrm{Arg}$. (C) Lactate was mainly produced in MM+Glu and MM+all. (D) $\mathrm{H}_{2} \mathrm{O}_{2}$ was produced in all groups, the lowest being in MM+Lac.

TABLE 1 | Changes (means \pm standard deviations, $n=3$ /group) in lactate concentration (mM) of $S$. mutans (Sm) after cultivation in the supernatant (Sup.) of S. oligofermentans (So) and L. reuteri (Lr).

\begin{tabular}{|c|c|c|c|c|}
\hline \multirow[t]{2}{*}{ Metabolite (mM) } & \multirow[t]{2}{*}{ Minimal medium } & \multicolumn{3}{|c|}{ Bacterial strain } \\
\hline & & $\mathrm{Sm}$ & Sm Sup. So ${ }^{A}$ & Sm Sup. Lr $^{B}$ \\
\hline \multirow[t]{6}{*}{ Lactate } & $\mathrm{MM}$ & $1.78 \pm 0.65 \times 10^{-2}$ & $-5.14 \pm 0.50 \times 10^{-2 \star \star \star}$ & $-1.13 \pm 1.08 \times 10^{-2 \star *}$ \\
\hline & $\mathrm{MM}+\mathrm{Glu}$ & $6.52 \pm 0.91$ & $3.84 \pm 0.40^{\star \star}$ & $2.95 \pm 0.31^{\star \star}$ \\
\hline & $\mathrm{MM}+\mathrm{Gly}+\mathrm{Glu}$ & $5.00 \pm 0.42$ & $3.01 \pm 0.49^{\star \star}$ & $3.65 \pm 0.33 \times 10^{-1 \star \star \star}$ \\
\hline & $\mathrm{MM}+\mathrm{Lac}+\mathrm{Glu}$ & $4.46 \pm 0.41$ & $-1.39 \pm 0.25^{\star \star \star}$ & $4.42 \pm 0.19$ \\
\hline & $\mathrm{MM}+\mathrm{Arg}+\mathrm{Glu}$ & $6.03 \pm 0.48$ & $1.69 \pm 0.43^{\star \star \star}$ & $3.29 \pm 0.26^{\star \star \star}$ \\
\hline & MM+all & $6.00 \pm 0.29$ & $-2.14 \pm 0.35^{\star \star \star}$ & $-0.46 \pm 0.11^{\star \star \star}$ \\
\hline
\end{tabular}

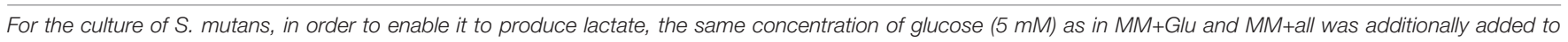

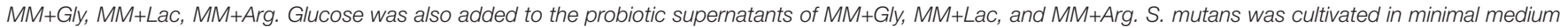

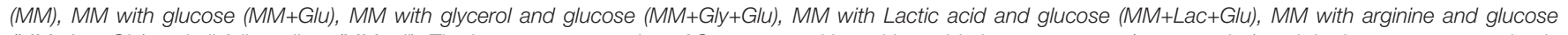

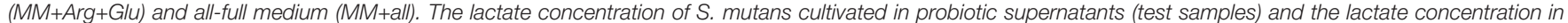

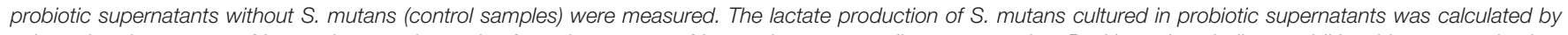

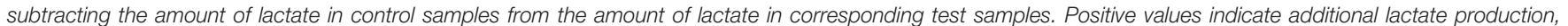
negative values indicate uptake of lactate by bacteria. Dunnett's test: ${ }^{\star} P<0.05,{ }^{* *} P<0.01,{ }^{* * *} P<0.001$, versus Sm. 


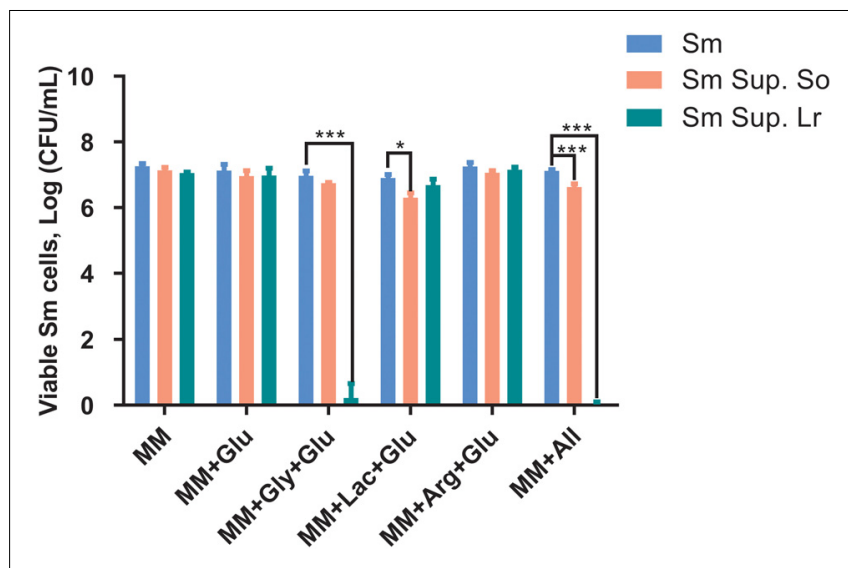

FIGURE 3 | Changes in colony-forming units (Log CFU/mL, means \pm standard deviations) of $S$. mutans after being cultured in the supernatant (Sup.) of S. oligofermentans (So) and L. reuteri (Lr). S. mutans was cultivated in minimal medium (MM), MM with glucose (MM+Glu), MM with glycerol and glucose (MM+Gly+Glu), MM with Lactic acid and glucose (MM+Lac+Glu), MM with arginine and glucose (MM+Arg+Glu) and all-full medium (MM+all) (controls), as well as supernatants of the probiotics. Glucose was additionally added to the MM+Gly, MM+Lac, and MM+Arg group of probiotic supernatants as it is described in section "Culture of S. mutans With Probiotic Supernatants." Each experiment was repeated three times. Dunnett's test: ${ }^{\star} P<0.05,{ }^{\star \star \star} P<0.001$, versus controls.

\section{Inhibitory Effect of the Probiotic Supernatants on the Survival of S. mutans}

When cultured in supernatants of $S$. oligofermentans and $L$. reuteri, survival of $S$. mutans was reduced compared with the controls (Figure 3). Specifically, treatment with the supernatant of $S$. oligofermentans resulted in a reduction in the viability of S. mutans in MM+Lac+Glu and MM+all by $0.6-\log _{10}$ and $0.5-\log _{10}$, respectively. Moreover, in MM+Gly+Glu and MM+all group, supernatant of $L$. reuteri yielded a reduction in viability of $6.1-\log _{10}$ and $7.1-\log _{10}$, respectively.

\section{DISCUSSION}

The present study investigated the potential of S. oligofermentans and $L$. reuteri to produce different antimicrobial substances under different environmental conditions, and tested the inhibitory effect of probiotic supernatants on lactate production and survival of $S$. mutans. We found that both probiotics produced $\mathrm{H}_{2} \mathrm{O}_{2}$ in different $\mathrm{MM}$, and ammonia in $\mathrm{MM}+\mathrm{Arg}$. In $\mathrm{MM}$ containing glycerol, $L$. reuteri also produced reuterin. Both probiotics produced lactate when glucose was present. Cultivation in the supernatants of both probiotics reduced the metabolic activity and survival of $S$. mutans significantly and environment-specifically. It can be assumed that these effects were not associated with $\mathrm{pH}$ changes (which were minimal given the buffering capacity of the used media), but associated with the products generated by the probiotics (Castillo et al., 2000). We hence confirm our hypothesis.
The mechanism by which probiotics precisely interfere with cariogenic pathogens remains unknown. There are currently three possible explanations, including (1) release of bacteriocins (Martinez et al., 2013), (2) the antimicrobial effects through co-aggregation (Lang et al., 2010), (3) competition with cariogenic bacteria for nutrition and adhesion (Terai et al., 2015; Schwendicke et al., 2017). In this study, S. mutans was cultured in the supernatant of probiotics only. Hence, we can make certain assumptions as to how these supernatants interacted with S. mutans: In MM+Lac and MM+all, S. oligofermentans had produced large amounts of $\mathrm{H}_{2} \mathrm{O}_{2}$, which has likely impacted on the survival and lactate production of $S$. mutans. In MM+Gly and $M M+a l l, L$. reuteri produced large amounts of reuterin, with potentially even more pronounced effects on $S$. mutans. The production of ammonia by $S$. oligofermentans in arginine containing medium had only moderate inhibitory effects on S. mutans activity and survival. Note that we cannot fully exclude the observed effects to be associated with other, non-measured probiotic products present in the supernatant, but given the consistency and plausibility of the measured presence of $\mathrm{H}_{2} \mathrm{O}_{2}$ and reuterin and the observed effects on $S$. mutans, the outlined pathway of how probiotics impact on $S$. mutans seems likely.

$\mathrm{H}_{2} \mathrm{O}_{2}$ is the major antibacterial substance produced by S. oligofermentans (Zhang et al., 2010; Tong et al., 2020), as confirmed by our study. S. oligofermentans possesses three $\mathrm{H}_{2} \mathrm{O}_{2}$-forming enzyme: lactate oxidase (Lox), that catalyzes L-lactate and oxygen to produce $\mathrm{H}_{2} \mathrm{O}_{2}$ and pyruvate; pyruvate oxidase (Pox), that generates $\mathrm{H}_{2} \mathrm{O}_{2}$ by oxidizing pyruvate to acetate via acetyl coenzyme; L-amino acid oxidase, that catalyzes the production of $\mathrm{H}_{2} \mathrm{O}_{2}$ from amino acids and peptone (Tong et al., 2008; Liu L. et al., 2012). Our findings that $\mathrm{H}_{2} \mathrm{O}_{2}$ production was most pronounced in $\mathrm{MM}+\mathrm{Lac}$ and $\mathrm{MM}+\mathrm{all}$, where lactic acid was available for Lox, suggest that Lox may play a role in $\mathrm{H}_{2} \mathrm{O}_{2}$ generation. The production of $\mathrm{H}_{2} \mathrm{O}_{2}$ in $\mathrm{MM}, \mathrm{MM}+\mathrm{Glu}, \mathrm{MM}+\mathrm{Gly}$ and $\mathrm{MM}+\mathrm{Arg}$ was similar, presumably because $S$. oligofermentans had converted extracellular glucose into intracellular polysaccharides during precultivation in $\mathrm{BHI}+$ glucose. When cultivated in carbohydrate-limited MM variations, intracellular glucose or glycogen of $S$. oligofermentans was decomposed into pyruvate. Pyruvate can generate $\mathrm{H}_{2} \mathrm{O}_{2}$ and acetyl phosphate through Pox or can be converted into lactic acid by the lactate dehydrogenase. The additional availability of glucose in MM+all may further support Pox and Lox activity, as $S$. oligofermentans converted glucose into pyruvate and lactate, which act as substrates for Pox and Lox, respectively. Overall, S. oligofermentans requires a Pox-Lox synergy to produce the maximum amount of $\mathrm{H}_{2} \mathrm{O}_{2}$ (Liu L. et al., 2012).

Saliva and protein-rich foods contain abundant amounts of arginine. The arginine deiminase system degrades and metabolizes arginine to ammonia (Nascimento, 2018), which raises the $\mathrm{pH}$ (Liu Y. L. et al., 2012). For S. oligofermentans, we found that ammonia can be detected in MM+Arg and $\mathrm{MM}+$ all. The lower ammonia production in $\mathrm{MM}+$ all may be explained by excess glucose being present, with the arginine deiminase activity decreasing when glucose 
concentrations exceed $2 \mathrm{mM}$ (Crow and Thomas, 1982; Kanapka and Kleinberg, 1983). Ammonia had only moderate effect on $S$. mutans activity and survival, which may be expected. Anti-caries effects of ammonia will be relevant nevertheless via altering the $\mathrm{pH}$ to a less cariogenic environment, hence supporting to rebalance remineralization over demineralization and preventing net mineral loss (Zaura and Twetman, 2019).

Reuterin is a multi-compound dynamic equilibrium system composed of 3-hydroxypropionaldehyde (3-HPA), its hydrate and dimer (Spinler et al., 2008) as well as acrolein. 3-HPA is a product of glycerol dehydration in the propanediol utilization (Pdu) pathway and is catalyzed by glycerol dehydratase (Chen and Hatti-Kaul, 2017). In reuterin solutions, acrolein and 3-HPA are interconverted, with acrolein being the active antimicrobial compound (Engels et al., 2016). In this study, we found $S$. oligofermentans to produce very little reuterin in $\mathrm{MM}+\mathrm{Gly}$ and $\mathrm{MM}+$ all. L. reuteri, however, is known to possess the Pdu pathway to produce reuterin via fermentation of glycerol. After cultivation in the reuterincontaining supernatant of $L$. reuteri, $S$. mutans survival was reduced nearly completely. Our results are in line with those from clinical studies finding $L$. reuteri to be an efficacious probiotic to combat oral pathogens (Lin et al., 2017; Geraldo et al., 2019).

It has also been shown that $L$. reuteri is capable of producing $\mathrm{H}_{2} \mathrm{O}_{2}$ (Kang et al., 2011; Basu et al., 2019), and in our experiments, $L$. reuteri produced $\mathrm{H}_{2} \mathrm{O}_{2}$ in each $\mathrm{MM}$ variation. $\mathrm{H}_{2} \mathrm{O}_{2}$ is mainly produced by Pox (as described) and NADH oxidase (Nox) (Hertzberger et al., 2014), while it is unclear which enzymatic pathway was relevant in our setting. It was found that Pox synthesis was inhibited when glucose was abundantly available, while Nox was not essentially affected (Sedewitz et al., 1984). This was not the case in $\mathrm{MM}+\mathrm{Glu}$ in our study. Hence, we assume that $\mathrm{H}_{2} \mathrm{O}_{2}$ was largely produced through NADHdependent reactions, as shown for Lactobacillus delbrueckii, too (Marty-Teysset et al., 2000).

To assess the influence of the products generated by the probiotics on the lactate production and survival of S. mutans, S. mutans was cultivated in the probiotic supernatants. When cultivated in $S$. oligofermentans supernatant, lactate production by $S$. mutans in each MM variation group was significantly reduced. Since the supernatants of each $\mathrm{MM}$ variation media contained $\mathrm{H}_{2} \mathrm{O}_{2}$, the reason for this decrease in lactate production may be related to $\mathrm{H}_{2} \mathrm{O}_{2}$. Furthermore, ammonia produced in $\mathrm{MM}+\mathrm{Arg}+\mathrm{Glu}$ and $\mathrm{MM}+$ all may also neutralize lactic acid produced by $S$. mutans, reminding us that adjustment the alkali-generation potential of oral microbial may also have great potential. A similar reduction in lactate production was found when S. mutans was cultured in $L$. reuteri supernatants for all groups except $\mathrm{MM}+\mathrm{Lac}+\mathrm{Glu}$. We consider that it is because the amount of $\mathrm{H}_{2} \mathrm{O}_{2}$ in $\mathrm{MM}+\mathrm{Lac}+\mathrm{Glu}$ was too small to reach an effective concentration.

Our findings agree with the study of Rossoni et al. (2018) in which they found the growth of S. mutans in planktonic cultures was inhibited by the bioactive substances released by Lactobacillus strains. In our study, almost no CFU were detectable in $\mathrm{MM}+\mathrm{Gly}+\mathrm{Glu}$ and $\mathrm{MM}+$ all of $\mathrm{L}$. reuteri supernatant, proving reuterin as a potentially powerful antibiotic substance. S. mutans showed significantly lower survival in the culture of the supernatants in $\mathrm{MM}+\mathrm{Lac}+\mathrm{Glu}$ and $\mathrm{MM}+$ all of S. oligofermentans, which indicated that large amounts of $\mathrm{H}_{2} \mathrm{O}_{2}$ produced in a lactate-rich environment may have an inhibitory effect on the survival of $S$. mutans.

Overall, our study demonstrated that the products generated by the probiotics in the supernatants may inhibit the metabolic activity and survival of $S$. mutans and this effect was environment-specific. While it may well be that additional benefits emerge from the usage of viable probiotic, e.g., via co-aggregation (probably between L. reuteri and S. mutans), competition with cariogenic bacteria for nutrients and adhesive surfaces, and isolation of substrate or metal ions, our findings open up new therapeutic avenues. Using supernatant or specific isolated compounds for inhibiting cariogenic pathogens comes with the advantage of being safer, easier to dose, and any product having an extended shelf-life compared with living probiotics. Understanding the interactions between probiotics and cariogenic bacteria in simulated oral environments and identifying the underlying molecular mechanisms may support more effective and safe applications.

This study has several limitations. First, the complex oral conditions cannot be completely simulated in vitro. The impact of other bacteria species and the relevance of further proteins being available for bacterial metabolization will likely modify our findings. For the sake of interpretability, however, a simplified model such as ours seems useful. Second, our method for detecting specific bacterial substances produced by probiotics using colorimetric assays was not comprehensive; a more detailed metabolomic analysis may yield further insights. Similar, determining the CFU/mL of $S$. mutans in planktonic bacterial cultures to assess survival inhibition comes with limitations and does not fully reflect that probiotic effects in a clinical setting should target dental biofilms. However, both the colorimetric assay and the enumeration of planktonic bacteria via $\mathrm{CFU} / \mathrm{mL}$ were chosen as they are easy to operate, reproducible and sufficient for the purposes of this study. Third, the culture time used in this study were optimized to capture, in a limited amount of time, the specific impact of the different metabolites. Different culture periods will be associated with different degrees of bacterial interaction. Last, there may be other mechanism, like end-product inhibition, as a possible non-specific mechanism that leads to a decrease in lactate production of $S$. mutans. However, the actual impact of this mechanism on our experimental results requires further verification.

In conclusion and within these limitations, the probiotic effects of S. oligofermentans and L. reuteri supernatants on the metabolic activity and survival of $S$. mutans were environmentspecific through different pathways. Future studies as well as clinical applications should consider environment-specific probiotic actions on cariogenic pathogens. 


\section{DATA AVAILABILITY STATEMENT}

All datasets generated for this study are included in the article/supplementary material.

\section{AUTHOR CONTRIBUTIONS}

HY performed the experiments, data analysis, and wrote the manuscript. PG and HY conceived and designed the study. PG guided the experiments and revised the manuscript. FS conceived the study, guided the design of the experiments, reviewed and edited the manuscript. All authors had approved the final version of the work.

\section{REFERENCES}

Aas, J. A., Paster, B. J., Stokes, L. N., Olsen, I., and Dewhirst, F. E. (2005). Defining the normal bacterial flora of the oral cavity. J. Clin. Microbiol. 43, 5721-5732. doi: 10.1128/JCM.43.11.5721-5732.2005

Bao, X., de Soet, J. J., Tong, H., Gao, X., He, L., van Loveren, C., et al. (2015). Streptococcus oligofermentans inhibits Streptococcus mutans in biofilms at both neutral pH and cariogenic conditions. PLoS One 10:e130962. doi: 10.1371/ journal.pone.0130962

Basu, T. P., Long, A. R., Nelson, B. J., Kumar, R., Rosenberg, A. F., and Gray, M. J. (2019). Complex responses to hydrogen peroxide and hypochlorous acid by the probiotic bacterium Lactobacillus reuteri. mSystems 4:e00453-19. doi: 10.1128/mSystems.00453-19

Bosch, M., Nart, J., Audivert, S., Bonachera, M. A., Alemany, A. S., Fuentes, M. C., et al. (2012). Isolation and characterization of probiotic strains for improving oral health. Arch. Oral Biol. 57, 539-549. doi: 10.1016/j.archoralbio.2011.10.006

Burne, R. A., and Marquis, R. E. (2000). Alkali production by oral bacteria and protection against dental caries. FEMS Microbiol. Lett. 193, 1-6. doi: 10.1016/ S0378-1097(00)00438-9

Castillo, A., Rubiano, S., Gutierrez, J., Hermoso, A., and Liebana, J. (2000). Post$\mathrm{pH}$ effect in oral streptococci. Clin. Microbiol. Infect. 6, 142-146. doi: 10.1046/j. 1469-0691.2000.00030.x

Chen, L., and Hatti-Kaul, R. (2017). Exploring Lactobacillus reuteri DSM20016 as a biocatalyst for transformation of longer chain 1,2-diols: limits with microcompartment. PLoS One 12:e185734. doi: 10.1371/journal.pone.0185734

Clancy, K. A., Pearson, S., Bowen, W. H., and Burne, R. A. (2000). Characterization of recombinant, ureolytic Streptococcus mutans demonstrates an inverse relationship between dental plaque ureolytic capacity and cariogenicity. Infect Immun. 68, 2621-2629. doi: 10.1128/iai.68.5.2621-2629.2000

Crow, V. L., and Thomas, T. D. (1982). Arginine metabolism in lactic streptococci. J. Bacteriol. 150, 1024-1032. doi: 10.1128/jb.150.3.1024-1032.1982

Doleyres, Y., Beck, P., Vollenweider, S., and Lacroix, C. (2005). Production of 3 hydroxypropionaldehyde using a two-step process with Lactobacillus reuteri. Appl. Microbiol. Biotechnol. 68, 467-474. doi: 10.1007/s00253-005-1895-4

Engels, C., Schwab, C., Zhang, J., Stevens, M. J. A., Bieri, C., Ebert, M. O., et al. (2016). Acrolein contributes strongly to antimicrobial and heterocyclic amine transformation activities of reuterin. Sci. Rep. 6:36246. doi: 10.1038/srep36246

Exterkate, R. A., Crielaard, W., and Ten, C. J. (2010). Different response to amine fluoride by Streptococcus mutans and polymicrobial biofilms in a novel highthroughput active attachment model. Caries Res. 44, 372-379. doi: 10.1159/ 000316541

Ganas, P., and Schwendicke, F. (2019). Effect of reduced nutritional supply on the metabolic activity and survival of cariogenic bacteriain in vitro. J. Oral Microbiol. 11:1605788. doi: 10.1080/20002297.2019.1605788

Ganzle, M. G., Holtzel, A., Walter, J., Jung, G., and Hammes, W. P. (2000). Characterization of reutericyclin produced by Lactobacillus reuteri LTH2584. Appl. Environ. Microbiol. 66, 4325-4333. doi: 10.1128/aem.66.10.4325-4333. 2000

\section{FUNDING}

This work was supported by the Deutsche Forschungsgemeinschaft (DFG SCHW 1766/2-2) and China Scholarship Council (CSC) (Grant \# 201708440325).

\section{ACKNOWLEDGMENTS}

We thank BioGaia company for kindly providing us with the Limosilactobacillus reuteri, ATCC PTA 5289 strain. We acknowledge support from the German Research Foundation (DFG) and the Open Access Publication Fund of Charite Universitätsmedizin Berlin.

Geraldo, B., Batalha, M. N., Milhan, N., Rossoni, R. D., Scorzoni, L., and Anbinder, A. L. (2019). Heat-killed Lactobacillus reuteri and cell-free culture supernatant have similar effects to viable probiotics during interaction with Porphyromonas gingivalis. J. Periodontal. Res. 55, 215-220. doi: 10.1111/jre.12704

Gruner, D., Paris, S., and Schwendicke, F. (2016). Probiotics for managing caries and periodontitis: systematic review and meta-analysis. J. Dent. 48, 16-25. doi: 10.1016/j.jdent.2016.03.002

Hertzberger, R., Arents, J., Dekker, H. L., Pridmore, R. D., Gysler, C., Kleerebezem, M., et al. (2014). $\mathrm{H}_{2} \mathrm{O}_{2}$ production in species of the lactobacillus acidophilus group: a central role for a novel NADH-dependent flavin reductase. Appl. Environ. Microbiol. 80, 2229-2239. doi: 10.1128/AEM.04272-13

Jalasvuori, H., Haukioja, A., and Tenovuo, J. (2012). Probiotic Lactobacillus reuteri strains ATCC PTA 5289 and ATCC 55730 differ in their cariogenic properties in vitro. Arch. Oral Biol. 57, 1633-1638. doi: 10.1016/j.archoralbio.2012.07.014

Jensen, A., Scholz, C., and Kilian, M. (2016). Re-evaluation of the taxonomy of the Mitis group of the genus Streptococcus based on whole genome phylogenetic analyses, and proposed reclassification of Streptococcus dentisani as Streptococcus oralis subsp. dentisani comb. nov., Streptococcus tigurinus as Streptococcus oralis subsp. tigurinus comb. nov., and Streptococcus oligofermentans as a later synonym of Streptococcus cristatus. Int. J. Syst. Evol. Microbiol. 66, 4803-4820. doi: 10.1099/ijsem.0.001433

Kanapka, J. A., and Kleinberg, I. (1983). Catabolism of arginine by the mixed bacteria in human salivary sediment under conditions of low and high glucose concentration. Arch. Oral Biol. 28, 1007-1015. doi: 10.1016/0003-9969(83) 90055-9

Kang, M., Oh, J., Lee, H., Lim, H., Lee, S., Yang, T.-H., et al. (2011). Inhibitory effect of Lactobacillus reuteri on periodontopathic and cariogenic bacteria. J. Microbiol. 49, 193-199. doi: 10.1007/s12275-011-0252-9

Lang, C., Bottner, M., Holz, C., Veen, M., Ryser, M., Reindl, A., et al. (2010). Specific Lactobacillus/mutans Streptococcus co-aggregation. J. Dent. Res. 89, 175-179. doi: 10.1177/0022034509356246

Lin, X., Chen, X., Tu, Y., Wang, S., and Chen, H. (2017). Effect of probiotic Lactobacilli on the growth of Streptococcus mutans and multispecies biofilms isolated from children with active caries. Med. Sci. Monit. 23, 4175-4181. doi: 10.12659/MSM.902237

Lin, X. B., Lohans, C. T., Duar, R., Zheng, J., Vederas, J. C., Walter, J., et al. (2015). Genetic determinants of reutericyclin biosynthesis in Lactobacillus reuteri. Appl. Environ. Microbiol. 81, 2032-2041. doi: 10.1128/AEM.03691-14

Liu, L., Tong, H., and Dong, X. (2012). Function of the pyruvate oxidaselactate oxidase cascade in interspecies competition between Streptococcus oligofermentans and Streptococcus mutans. Appl. Environ. Microbiol. 78, 2120 2127. doi: 10.1128/AEM.07539-11

Liu, Y., Chu, L., Wu, F., Guo, L., Li, M., Wang, Y., et al. (2014). Influence of pH on inhibition of Streptococcus mutans by Streptococcus oligofermentans. Eur. J. Oral Sci. 122, 57-61. doi: 10.1111/eos.12102

Liu, Y. L., Nascimento, M., and Burne, R. A. (2012). Progress toward understanding the contribution of alkali generation in dental biofilms to inhibition of dental caries. Int. J. Oral Sci. 4, 135-140. doi: 10.1038/ijos.2012.54 
Marsh, P. D. (2006). Dental plaque as a biofilm and a microbial community implications for health and disease. BMC Oral Health 6(Suppl 1):S14. doi: 10.1186/1472-6831-6-S1-S14

Martinez, F. A., Balciunas, E. M., Converti, A., Cotter, P. D., and de Souza, O. R. (2013). Bacteriocin production by Bifidobacterium spp. a review. Biotechnol. Adv. 31, 482-488. doi: 10.1016/j.biotechadv.2013.01.010

Marty-Teysset, C., de la Torre, F., and Garel, J. (2000). Increased production of hydrogen peroxide by Lactobacillus delbrueckii subsp. bulgaricus upon aeration: involvement of an NADH oxidase in oxidative stress. Appl. Environ. Microbiol. 66, 262-267. doi: 10.1128/aem.66.1.262-267.2000

Meurman, J. H. (2005). Probiotics: do they have a role in oral medicine and dentistry? Eur. J. Oral Sci. 113, 188-196. doi: 10.1111/j.1600-0722.2005.00191.x

$\mathrm{Mu}$, Q., Tavella, V. J., and Luo, X. M. (2018). Role of Lactobacillus reuteri in human health and diseases. Front. Microbiol. 9:757. doi: 10.3389/fmicb.2018.00757

Nascimento, M. M. (2018). Potential uses of arginine in dentistry. Adv. Dent. Res. 29, 98-103. doi: 10.1177/0022034517735294

Ng, S. C., Hart, A. L., Kamm, M. A., Stagg, A. J., and Knight, S. C. (2009). Mechanisms of action of probiotics: recent advances. Inflamm. Bowel Dis. 15, 300-310. doi: 10.1002/ibd.20602

Rossoni, R. D., Velloso, M. D. S., de Barros, P. P., de Alvarenga, J. A., Santos, J. D. D., Prado, A. C. C. D. S., et al. (2018). Inhibitory effect of probiotic Lactobacillus supernatants from the oral cavity on Streptococcus mutans biofilms. Microb. Pathog. 123, 361-367. doi: 10.1016/j.micpath.2018.07.032

Schwendicke, F., Korte, F., Dörfer, C. E., Kneist, S., Fawzy El-Sayed, K., and Paris, S. (2017). Inhibition of Streptococcus mutans growth and biofilm formation by probiotics in vitro. Caries Res. 51, 87-95. doi: 10.1159/000452960

Sedewitz, B., Schleifer, K. H., and Gotz, F. (1984). Physiological role of pyruvate oxidase in the aerobic metabolism of Lactobacillus plantarum. J. Bacteriol. 160, 462-465. doi: 10.1128/jb.160.1.462-465.1984

Spinler, J. K., Taweechotipatr, M., Rognerud, C. L., Ou, C. N., Tumwasorn, S., and Versalovic, J. (2008). Human-derived probiotic Lactobacillus reuteri demonstrate antimicrobial activities targeting diverse enteric bacterial pathogens. Anaerobe 14, 166-171. doi: 10.1016/j.anaerobe.2008.02.001

Talarico, T. L., and Dobrogosz, W. J. (1989). Chemical characterization of an antimicrobial substance produced by Lactobacillus reuteri. Antimicrob. Agents Chemother. 33, 674-679. doi: 10.1128/aac.33.5.674

Terai, T., Okumura, T., Imai, S., Nakao, M., Yamaji, K., Ito, M., et al. (2015). Screening of probiotic candidates in human oral bacteria for the prevention of dental disease. PLoS One 10:e128657. doi: 10.1371/journal.pone.012 8657
Tong, H. (2003). Streptococcus oligofermentans sp. nov., a novel oral isolate from caries-free humans. Int. J. Syst. Evol. Microbiol. 53, 1101-1104. doi: 10.1099/ijs. 0.02493-0

Tong, H., Chen, W., Merritt, J., Qi, F., Shi, W., and Dong, X. (2007). Streptococcus oligofermentans inhibits Streptococcus mutans through conversion of lactic acid into inhibitory $\mathrm{H}_{2} \mathrm{O}_{2}$ : a possible counteroffensive strategy for interspecies competition. Mol. Microbiol. 63, 872-880. doi: 10.1111/j.1365-2958.2006. 05546.x

Tong, H., Chen, W., Shi, W., Qi, F., and Dong, X. (2008). SO-LAAO, a novel L-amino acid oxidase that enables Streptococcus oligofermentans to outcompete Streptococcus mutans by generating $\mathrm{H}_{2} \mathrm{O}_{2}$ from peptone. J. Bacteriol. 190, 4716-4721. doi: 10.1128/JB.00363-08

Tong, H., Dong, Y., Wang, X., Hu, Q., Yang, F., Yi, M., et al. (2020). Redoxregulated adaptation of Streptococcus oligofermentans to hydrogen peroxide stress. mSystems 5:e00006-20. doi: 10.1128/mSystems.00006-20

Wong, L., and Sissions, C. H. (2001). A comparison of human dental plaque microcosm biofilms grown in an undefined medium and a chemically defined artificial saliva. Arch. Oral Biol. 46, 477-486. doi: 10.1016/S0003-9969(01) 00016-4

Zaura, E., and Twetman, S. (2019). Critical appraisal of oral pre- and probiotics for caries prevention and care. Caries Res. 53, 514-526. doi: 10.1159/000499037

Zhang, J., Tong, H. C., Dong, X. Z., Yue, L., and Gao, X. J. (2010). A preliminary study of biological characteristics of Streptococcus oligofermentans in oral microecology. Caries Res. 44, 345-348. doi: 10.1159/000315277

Zheng, J., Wittouck, S., Salvetti, E., Franz, C., Harris, H., Mattarelli, P., et al. (2020). A taxonomic note on the genus Lactobacillus: description of 23 novel genera, emended description of the genus Lactobacillus Beijerinck 1901, and union of Lactobacillaceae and Leuconostocaceae. Int. J. Syst. Evol. Microbiol. 70:4107. doi: 10.1099/ijsem.0.004107

Conflict of Interest: The authors declare that the research was conducted in the absence of any commercial or financial relationships that could be construed as a potential conflict of interest.

Copyright (c) $2020 \mathrm{Yu}$, Ganas and Schwendicke. This is an open-access article distributed under the terms of the Creative Commons Attribution License (CC BY). The use, distribution or reproduction in other forums is permitted, provided the original author(s) and the copyright owner(s) are credited and that the original publication in this journal is cited, in accordance with accepted academic practice. No use, distribution or reproduction is permitted which does not comply with these terms. 